\section{PWE-139 FURTHER VALIDATION OF TERMINAL PEPTIDE OF PROCOLLAGEN III (PIIINP) FOR THE DETECTION AND ASSESSMENT OF NONALCOHOLIC STEATOHEPATITIS IN PATIENTS WITH NONALCOHOLIC FATTY LIVER DISEASE}

doi:10.1136/gutjnl-2013-304907.427

1,"S Tanwar, ${ }^{2} \mathrm{~J}$ Parkes, ${ }^{1} \mathrm{~B}$ J Hogan, ${ }^{3} \mathrm{D}$ Schuppan, 'M Pinzani, ${ }^{4} \mathrm{M}$ J P Arthur, ${ }^{5} \mathrm{~A}$ Burt, 'W M Rosenberg. 'UCL Institute for Liver \& Digestive Health, University College London, London; ${ }^{2}$ Public Health Sciences \& Medical Statistics, Faculty of Medicine, University of Southampton, Southampton, UK; ${ }^{3}$ University of Mainz, Mainz, Germany; ${ }^{4}$ University of Leeds, Leeds; ${ }^{5}$ Institute of Cellular Medicine, Newcastle University, Newcastle upon Tyne, UK

Introduction PIIINP has recently been shown to discriminate between simple steatosis (SS) and NASH both in patients without advanced fibrosis and in patients with all degrees of fibrosis ${ }^{1}$. In this study we validated PIIINP as a biomarker of NASH in a cohort of patients with biopsy proven NAFLD and evaluated its performance at the proposed diagnostic thresholds.

Methods 71 patients with NAFLD and no evidence of other liver disease were included in this study. Liver biopsies were performed on all patients and analysed by a expert liver histopathologist. All liver biopsies were of suitable size for analysis $(>12 \mathrm{~mm}$ and $>5$ portal tracts) and classified in a dichotomous manner into those with SS or histological NASH. Fibrosis was assessed using the Scheuer classification. Serum samples were taken at the time of liver biopsy.

Results 14 of the 60 patients with non-advanced fibrosis (4-F0, 18-F1.1-F2) and all 11 patients with advanced fibrosis (9-F3, 2-F4) had NASH respectively. The AUROC of PIIINP in discriminating between SS and NASH in patients with non-advanced fibrosis and all degrees of fibrosis was 0.81 (CI 0.69-0.94) and 0.87 (CI $0.79-0.96)$ respectively. In comparison, the ability of ALT to discriminate between SS and NASH ranged between $0.43-0.45$. The performance of the recently proposed thresholds PIIINP in their ability to diagnose NASH in our population is displayed in the table.

\begin{tabular}{lll}
\multicolumn{3}{l}{ Abstract PWE-139 Table } \\
\hline \begin{tabular}{lll}
\hline PIIINP Threshold & F0-2: & F0-4: \\
ng/ml & NPV/PPV \% & NPV/PPV \% \\
\hline 5.2 & $92 / 42$ & $88 / 56$ \\
7.2 & $85 / 68$ & $83 / 85$ \\
11 & $77 / 100$ & $72 / 100$ \\
\hline
\end{tabular}
\end{tabular}

Conclusion PIIINP discriminates between SS and NASH. The performance of the proposed diagnostic thresholds is comparable to that reported in the original publication of this biomarker. Our results suggest that PIIINP can be used to detect the minority of patients with NAFLD who have NASH and are at risk of developing progressive fibrosis.

Disclosure of Interest None Declared.

\section{REFERENCE}

1. Tanwar S et al. Validation of Terminal Peptide of Procollagen III for the Detection and Assessment of Nonalcoholic Steatohepatitis in Patients with Nonalcoholic Fatty Liver Disease. Hepatology. 2013 Jan; 57(1):103-11. doi: 10.1002/hep.26030. PMID: 22930399

\section{PWE-140 COMPARISON OF 4 SERUM MARKERS PANELS OF FIBROSIS IN CHC: VARIANTS OF THE HYALURONIC ACID (HA) ASSAY SIGNIFICANTLY AFFECT THEIR DIAGNOSTIC PERFORMANCE}

doi:10.1136/gutjnl-2013-304907.428
1.*S Tanwar, 'P M Trembling, 'B Hogan, ${ }^{2} \mathrm{E} L$ Ellis, ${ }^{2} \mathrm{~J}$ Parkes, ${ }^{3} \mathrm{P}$ Grant, ${ }^{3} \mathrm{E}$ Nastouli, ${ }^{4} \mathrm{C}$ Herold, ${ }^{5} \mathrm{D}$ Schuppan, 'W M Rosenberg. 'UCL Institute for Liver \& Digestive Health, University College London, London; 'University of Southampton, Southampton; ${ }^{3}$ Department of Clinical Microbiology and Virology, University College London Hospitals NHS Foundation Trust, London, UK; ${ }^{4}$ University of Erlangen, Erlangen; ${ }^{5}$ Molecular and Translational Medicine, Department of Medicine, University of Mainz, Mainz, Germany

Introduction The detection of advancing fibrosis in patients with $\mathrm{CHC}$ and prior treatment failure is important for ascertaining prognosis. HA has been used alone and as a constituent component of fibrosis marker panels. The aim of this study was to compare the performance of 4 marker panels in the detection of moderate-tosevere fibrosis (Metavir F2-4) and to assess the influence on diagnostic performance of using 2 different validated assays for HA.

Methods 80 patients with CHC, all non-responders or relapsers to IFN-based treatment, were included in this study. Sera obtained within 6 months of liver biopsy were used to measure 4 biomarker panels incorporating HA (ELF, Fibrospect-II, Hepascore, Fibrometer2G) using 2 validated assays for HA (ELISA-Siemens, radiometricPharmacia). Diagnostic performance for the detection of moderate-to-severe fibrosis was assessed by AUROC and by evaluating biomarker performance at published thresholds.

Results The prevalence of moderate-to-severe fibrosis was 63\% (F0-8\%, F1-29\%, F2-24\%, F3-30\%, F4-9\%). The AUROC of the Siemens HA assay was higher than the Pharmacia assay $(0.80 \mathrm{Vs}$. $0.69, \mathrm{P}=0.005)$. Incorporating the Siemens assay for HA, the performance of the panels were not statistically significantly different but Fibrometer 2G generated the highest AUROC. Using the Siemens assay, ELF and Fibrometer $2 \mathrm{G}$ had the highest PPV and NPV respectively (88\% and $100 \%$ using published thresholds). The use of the Pharmacia assay for HA to calculate the biomarkers did not reduce the discriminatory ability of the 4 panels $(p=N S)$. However whereas the other panels were largely unaffected, the use of the Pharmacia assay resulted in a dramatic reduction in the performance of published thresholds of the ELF test with the a prioriand a posteriori probability of fibrosis being equal.

\section{Abstract PWE-140 Table}

\begin{tabular}{lll}
\hline & $\begin{array}{l}\text { HA-Siemens } \\
\text { AUROC } \\
\text { (PPV/NPV) }\end{array}$ & $\begin{array}{l}\text { HA-Pharmacia } \\
\text { AUROC } \\
\text { (PPV/NPV) }\end{array}$ \\
\hline HEPASCORE & $0.85(79 / 67)$ & $0.81(73 / 72)$ \\
FIBROMETER2G & $0.88(71 / 100)$ & $0.87(70 / 100)$ \\
ELF & $0.84(88 / 62)$ & $0.79(63 / 37)$ \\
FIBROSPECTII & $0.84(86 / 59)$ & $0.81(84 / 55)$ \\
\hline
\end{tabular}

Conclusion In this study the performance of the 4 biomarker panels to detect moderate-to-severe fibrosis was comparable. The diagnostic performance of biomarker panels may be significantly effected by the selection of the individual component assays as demonstrated by comparison of the results obtained with different HA assays.

Disclosure of Interest None Declared.

\section{PWE-141 CONTINUITY OF CARE IN HEPATITIS C PATIENTS SERVING A CUSTODIAL SENTENCE IN SCOTLAND}

doi:10.1136/gutjnl-2013-304907.429

1,"S A Smith, ${ }^{2} \mathrm{~J}$ Dillon, ${ }^{3}$ S Fraser. 'Palliative Medicine, Queen Elizabeth Hospital, Newcastle, UK; ${ }^{2}$ Gastroenterology, Ninewells Hospital, Dundee, -; 3 Public Health, HMP Perth/HMP Open Estate, Dundee, UK

Introduction Hepatitis C Virus (HCV) has been deemed by the Scottish Government to be 'One of the most serious and significant public health risks of our generation'. ${ }^{1}$ Approximately $90 \%$ of those who are currently infected acquired the virus through drug injecting 